\title{
Depurando la Terminologia Anatomica: Apertura y Foramen, Dos Similitudes y Redundancia Terminológica
}

\author{
Refining the Anatomical Terminology: Opening and Foramen, Two Similarities and Redundancy
}

\author{
*,***orge Eduardo Duque Parra; *John Barco Rios \& ${ }^{* * *}$ Natalia Duque Quintero
}

DUQUE PARRA, J. E.; BARCO, R. J. \& DUQUE, Q. N. Depurando la Terminologia Anatomica: apertura y forámen, dos similitudes y redundancia terminológica. Int. J. Morphol., 30(4):1497-1499, 2012.

RESUMEN: El uso redundante de términos en el campo de la morfología, además de dificultar el aprendizaje de los estudiantes, va en contra de los preceptos establecidos por el Programa Federativo Internacional para la Terminología Anatómica (FIPAT) sobre el uso de una terminología anatómica unificada, mediante la cual se pretende evitar la redundancia terminológica. Se propone suprimir el término apertura piriforme y reemplazarlo por el término foramen piriforme, con el fin de evitar la ambigüedad y la utilización de distintos nombres que describen a una estructura de similares características.

PALABRAS CLAVE: Apertura; Foramen; Terminología.

\section{INTRODUCCIÓN}

"Quien no conoce bien la fuerza de las palabras no puede conocer bien a los hombres" (Confucio).

El término apertura deriva del término latín aperire que significa abrir, palabra utilizada para designar un orificio (Dorland, 2005), abertura o agujero (Braier, 1980; Dorland, 2005; García de Diego, 2000; Soukhanov, 1992), este último término se acostumbra utilizar como sinónimo (Diccionario Terminológico de Ciencias Médicas, 1983), se trata de una palabra que deriva de la voz aguja, la cual proviene del latín acúcula que denota una abertura redondeada donde la partícula ac da la idea de penetrante (Del Hovo et al., 1977). El término foramen es otra palabra latina utilizada para designar un agujero, orificio o abertura (García de Diego; Diccionario Terminológico de Ciencias Médicas; Dorland, 1994).

La apertura piriforme es un orificio con forma de pera (Dauber, 2007), el cual describe aquel espacio delimitado por las maxilas y los huesos nasales y que corresponde al orificio de entrada a la cavidad nasal (Moore \& Dalley, 2007; Coello \& Coello, 2009) contribuyendo a la formación de la nariz, cuya morfología varía de acuerdo a los grupos étnicos y al sexo (Prado et al., 2011). En la Terminologia
Anatomica, el término "apertura" aparece relacionado en la norma facial como un componente del cráneo, sólo y específicamente para la apertura piriforme [A02.100.088] (FICAT, 1998) (ver Fig. 1).

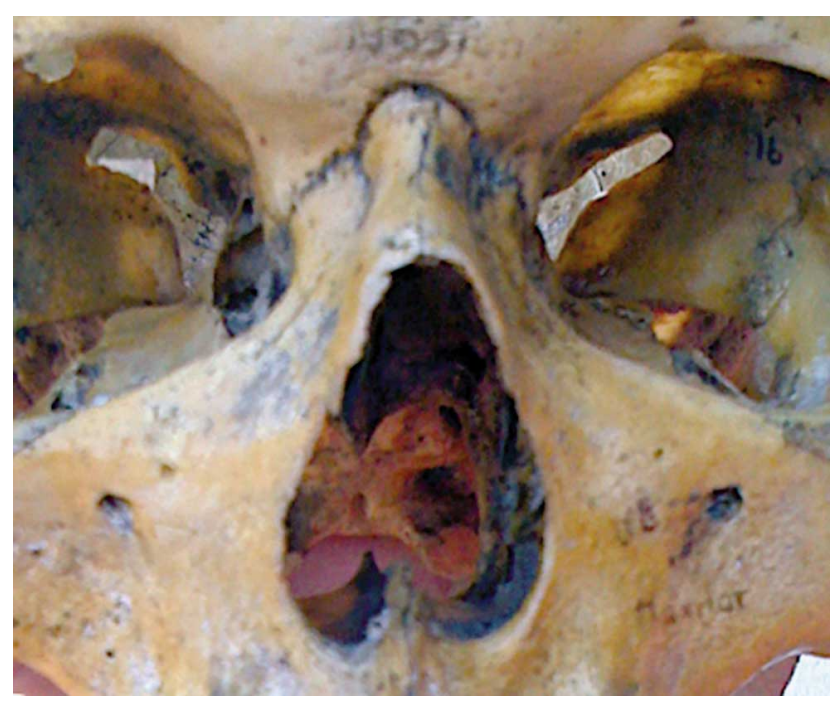

Fig. 1. Norma facial del cráneo que resalta el foramen piriforme en la región central.

\footnotetext{
* Departamento de Ciencias Básicas, Programa de Medicina, Universidad de Caldas, Manizales, Colombia.

** Departamento de Ciencias Básicas Biológicas, Universidad Autónoma de Manizales, Manizales, Colombia.

****Departamento de Antropología, Universidad de Caldas, Manizales, Colombia.
} 


\section{DISCUSIÓN}

La terminología médica, que incluye la Terminologia Anatomica, se encuentra referida en diccionarios terminológicos en los que están descritos entre cuarenta mil y cien mil vocablos (en la que se incluye la anatomía macroscópica), de los cuales los estudiantes de Medicina del área pre-clínica deben aprender alrededor de quince mil de ellos. Este lenguaje es internacional y no incluye vocablos comunes, pues con estos es prácticamente imposible referirse con precisión y de forma concisa a un significado (López Piñero \& Terrada Ferrandis, 1990), incluso morfológico.

A finales del siglo XIX existían más de cincuenta mil términos anatómicos que se aplicaban sólo a cinco mil o seis mil estructuras del cuerpo humano (López Piñero \& Terrada Ferrandis; Hollinshead, 1983), con un promedio de diez nombres por estructura (Hollinshead), lo que significaba una alta repetición de términos por la utilización de sinónimos y de epónimos. Debido a ello, en 1895, los anatomistas alemanes prepararon y publicaron en Basilea una lista en la que se incluían sólo cuatro mil quinientos términos aproximadamente, conocida como Nomina Anatomica de Basilea (B.N.A) (Chatain, 1967; Hollinshead; O’Rahilly, 1989), la cual tenía como propósito unificar la terminología anatómica y facilitar la comunicación entre la comunidad de anatomistas (Cárdenas et al., 2010). Dicha nómina fue ratificada en el primer Congreso Internacional de Anatomía celebrado en Ginebra. Ya en París, durante el VI Congreso Internacional realizado en 1955, se llegó a un acuerdo internacional sobre la adopción de un sistema de terminología en latín, basado en gran parte en la nomenclatura de Basilea, de donde surge entonces la Nomina Anatomica de París (P.N.A.) (O’Rahilly), de la cual se deriva la presente Terminología Anatómica.

Aunque la terminología médica actual es el sedimento de unos veinticinco siglos de medicina científica (López Piñero \& Terrada Ferrandis), ésta se ha ido depurando con el paso del tiempo para asegurar que las descripciones no sean ambiguas (Moore \& Dalley). Por lo tanto, toda palabra que surge inicialmente como un término ocasional y transitorio puede sucederle una de dos cosas, configurarse sucesivamente y perpetuarse o morir por desuso (Laín Entralgo, 1994). El desuso de algunos términos puede deberse a su escasa o nula descripción para una correcta comunicación entre las comunidades académicas, que en el caso morfológico es determinado por la Federación Internacional de Anatomistas (IFA), y son analizados por morfólogos de distintas nacionalidades, como actualmente se hace en los Simposios Ibero-latinoamericanos de Terminología
(SILAT) y organizados por la Asociación Panamericana de Anatomía (Cruz et al., 2010). Estos SILAT se realizan periódicamente desde el 2009 y tienen como objeto lograr una mejor precisión y claridad en la denominación de las diversas estructuras del cuerpo humano (Losardo et al., 2010), pues es infortunado que una carencia de uniformidad y de su uso en países o zonas de un mismo país, lleve a desconocimiento de lo que dicen los otros (Duque Parra et al., 2002).

El vocabulario grecolatino continúa siendo el principal idioma para la nominación de los distintos términos empleados en las ciencias médicas, pero la ausencia de un criterio uniforme supone serias dificultades que el lenguaje tiene que asumir como instrumento fundamental de comunicación entre todos los que integran la comunidad médica internacional (López Piñero \& Terrada Ferrandis), incluida la terminología anatómica. Debido a esa heterogeneidad de términos empleados es que surge la necesidad de implementar una terminología estándar (Hollinshead; Chatain; Lippert, 2005). No obstante, a pesar de los logros alcanzados, aun se presentan controversias y desacuerdos frente a la persistencia de algunos términos redundantes (Whitmore, 1999), los que podrían ser obviados con el uso de una terminología anatómica más simplificada. Tal es el caso de los términos "apertura" y "foramen", los cuales resultan ser sinónimos y redundantes, pues son dos términos utilizados para referirse a una misma cosa. Además, el término "apertura" aparece una sola vez en toda la terminología anatómica, por lo que podría ser considerada como una palabra en desuso, lo cual no sucede con el término "foramen" que sí es utilizado frecuentemente.

En concordancia con lo anterior y de acuerdo con el objetivo promulgado en el simposio Ibero-Latinoamericano de Terminologia Anatomica, que es el ente encargado de revisar, discutir y sugerir modificaciones de la terminología vigente para ser presentadas al Comité Internacional de Terminología Anatómica (Terminologia Anatomica, 2010) para su aprobación o desaprobación, sometemos a consideración éste análisis sobre la "apertura piriforme".

\section{CONCLUSIÓN}

La Terminologia Anatomica debe seguir depurándose racionalmente con el propósito de evitar la utilización innecesaria de términos sinónimos que describen a una misma estructura, por lo que se sugiere suprimir el término apertura y ser reemplazado por el término foramen. Por tanto, la apertura piriforme debería ser nominada como foramen piriforme. 
DUQUE PARRA, J. E.; BARCO, R. J. \& DUQUE, Q. N. Refining the Anatomical Terminology: opening and foramen. two similarities and redundancy. Int. J. Morphol., 30(4):1497-1499, 2012.

SUMMARY: The redundant use of terms in morphology field, as well as hindering the learning of students, goes against the precepts established long ago by the Federative International Programme for Anatomical Terminology (FIPAT) on the use of unified anatomical terminology, which is meant to avoid the redundancy terms. It is proposed to delete the term piriform aperture and replace it for the term piriform foramen in order to avoid ambiguity and the use of different names that describe similar structure features.

KEY WORDS: Foramen; Opening; Terminology.

\section{REFERENCIAS BIBLIOGRÁFICAS}

Braier, L. Diccionario enciclopédico de Medicina JIMS. Barcelona, Editorial JIMS, 1980.

Cárdenas, J.; Rodríguez, A. \& Godoy, K. ¿Enseñanza de la terminología en la enseñanza media en Chile? Relato de una experiencia. Int. J. Morphol., 28(4):1366, 2010.

Chatain, I. Terminología Anatómica. Cali, Editorial Norma, 1967.

Coello, C. R. \& Coello, S. R. Anatomía Humana. Descripción por regiones. Guayaquil, Universidad de Guayaquil, 2009.

Cruz, G. R.; Rodríguez, T. A.; Prates, J. C.; Losardo, R. J. \& Prates, N. E. V. R. Simposio Ibero-Latinoamericano de Terminología morfológica. Características generales. Int. J. Morphol., 28(2):643-6, 2010.

Dauber, W. Pocket Atlas of Human Anatomy. New York, Thieme, 2007.

Del Hovo, R. C.; López Chávez, J. \& Pimentel Álvarez, J. La fuerza de las palabras. México, Reader's Digest S. A. de C. V., 1977.

Diccionario Terminológico de Ciencias Médicas. Barcelona, Salvat Editores S. A., 1983.

Dorland, B. Dorland diccionario enciclopédico ilustrado de Medicina. Madrid, Elsevier, 2005.

Dorland, W. A. Dorland's illustrated medical dictionary. $28^{\text {th }}$ ed. Philadelphia, W. B. Saunders Company, 1994.

Duque Parra, J. E.; Gómez Arias, N. C. \& Giraldo Ríos, D. P. Nomenclatura anatómica internacional: un horno microondas en el interior de un volcán activo?. Medicina UPB, 21(1):43-55, 2002 .

Federative International Committee on Anatomical Terminologies (FICAT). Terminologia Anatomica. International Anatomical Terminology. New York, Thieme, 1998.

García de Diego, V. Diccionario Ilustrado Latino-Español Español-Latino. Barcelona, Spes, 2000.
Hollinshead, W. H. Anatomía para cirujanos dentistas. México, Harla y Harper \& Row Latinoamericana, 1983.

Laín Entralgo, P. Lenguaje medico: desordenes genéticos. Perfiles, 2:2, 1994.

Lippert, H. Anatomía texto y atlas. Madrid, Marbán Libros S. L., 2005.

López Piñero, J. M. \& Terrada Ferrandis, M. L. Introducción a la terminología médica. Barcelona, Salvat Editores S. A., 1990.

Losardo, R. J.; Cruz Gutiérrez, R; Rodríguez Torres, A.; Prates, J. C. $\&$ Prates, N. E. V. R. Simposios Ibero Latinoamericanos de Terminología morfológica (SILAT). Primeros dos años y normativas vigentes. Int. J. Morphol., 28(4):1323-6, 2010.

Moore, K. L. \& Dalley, A. F. Anatomía con orientación clínica. Buenos Aires, Médica Panamericana, 2007.

O`Rahilly, R. Anatomía de Gardner. 5ª ed. Mexico D. F., Interamericana McGraw-Hill, 1989.

Prado, F. B.; Caldas, R. A.; Rossi, A. C.; Freire, A. R.; Groppo, F .C.; Caria, P. H. F. \& Junior, E. D. Piriform apertura morphometry and nasal bones morphology in Brazilian population by posteroanterior Caldwell radiographys. Int. J. Morphol., 29(2):393-8, 2011.

Soukhanov, A. H. The American heritage dictionary of the english language. Boston, Houghton Mifflin Company, 1992.

Terminologia Anatomica. Jornal da USP, 903:23, 2010.

Whitmore, I. Terminologia Anatomica: new terminology for the new anatomist. Anat. Rec., 257(2):50-3, 1999.

Dirección para Correspondencia:

Jorge Eduardo Duque Parra

Departamento de Ciencias Básicas

Programa de Medicina, Facultad de Ciencias para la Salud. Universidad de Caldas

Manizales

COLOMBIA

Recibido : 06-11-2011

Aceptado: 11-05-2012

Email: jorge.duque_p@ucaldas.edu.co 